\title{
Antimicrobial Property of the Newly Formulated Health Drink Against Common Oral Pathogen, Streptococcus Mutans
}

\author{
P. Keshaav Krishnaa ${ }^{1}$, V. Vishnu Priya*2, R. Gayathri ${ }^{3}$ and R. V. Geetha ${ }^{4}$ \\ ${ }^{1}$ Saveetha Dental College,Saveetha Institute of Medical and Technical Sciences, \\ Saveetha University,Chennai-77, India \\ ${ }^{2}$ Professor Department of Biochemistry, Saveetha Dental College,Saveetha Institute \\ of Medical and Technical Sciences, Saveetha University,Chennai-77, India \\ ${ }^{3}$ Assistant Professor Department of Biochemistry, Saveetha Dental College,Saveetha \\ Institute of Medical and Technical Sciences, Saveetha University,Chennai-77, India \\ ${ }^{4}$ Associate Professor Department of Microbiology, Saveetha Dental College,Saveetha \\ Institute of Medical and Technical Sciences, Saveetha University,Chennai-77, India
}

\begin{abstract}
An important property that is necessary in a health drink is to have good antimicrobial property. This is a first of its kind health drink that focuses primarily on oral health. The aim of the present study was to estimate the antimicrobial activity of a newly formulated health drink. The various components were weighed out and grinded to fine homogeneous powder.The sample health drink powder was prepared by weighing out $10 \mathrm{~g}$ of whole black pepper, $18 \mathrm{~g}$ of almonds, $15 \mathrm{~g}$ of raisins, $10 \mathrm{~g}$ of dried and powdered mango peel and fruit along with about $1 \mathrm{~g}$ of turmeric powder. The same was then assessed against Streptococcus mutans by incorporating it in different dilutions in nutrient agar and incubating the same. It was observed that there was reduction in the growth with increase in concentration. The final growth cultures were compared with the positive and negative controls to estimate the efficacy of the health drink. When colonies were counted it was observed that there was a significant reduction in colonies between $200 \mathrm{ml}$ of the extract and $1000 \mathrm{ml}$ of the extract. This was a very significant finding as the usage of the health drink would in turn potentially decrease the microbial load in the oral cavity. Within the limits of the present study the formulated health drink proves to have a very good antibacterial activity against common oral pathogens and the discussion also proves the efficacy of the same against systemic pathogens as well. Further studies would lead to the introduction of a superior health drink in the market which would help the entire human race.
\end{abstract}

KEY WORDS: NEWLY FORMULATED HEALTH DRINK, ANTIMICROBIAL, ORAL HEALTH DRINK, HERBAL SUPPLEMENT.

\section{ARTICLE INFORMATION}

${ }^{*}$ Corresponding Author: vishnupriya@saveetha.com

Received 27th July 2020 Accepted after revision 20th Sep 2020

Print ISSN: 0974-6455 Online ISSN: 2321-4007 CODEN: BBRCBA

Thomson Reuters ISI Web of Science Clarivate Analytics USA and Crossref Indexed Journal

$$
\begin{aligned}
& \text { Clarivate } \\
& \text { Analytics }
\end{aligned}
$$




\section{INTRODUCTION}

Dental caries, otherwise known as tooth decay, is one of the most prevalent chronic diseases of people worldwide; individuals are susceptible to this disease throughout their lifetime. Dental caries forms through a complex interaction over time between acid-producing bacteria and fermentable carbohydrate, and many host factors including teeth and saliva. The disease develops in both the crowns and roots of teeth, and it can arise in early childhood as an aggressive tooth decay that affects the primary teeth of infants and toddlers. There is no particular health drink that focuses on oral health, various focus on systemic health.

Initially the constituents of tea were studied as it is one of the most drinks that are consumed in the population. and the effect of each compound was observed. Literature evidence showed that the major component of tea was Polyphonic compounds. Among the polyphonic compounds the major compounds were flavonoids. Within flavonoids the major one was tannins (Glade, 2000). When Tannins undergo oxidation produced theaflavins and thearubigins which were responsible for the dark colour and robust flavour of tea. Other major flavonoids that were present were Catechin, epicatechin, epicatechin gallate, gallocatchin, epigallocatechin gallate (EGCG) (Knop et al., 2015). EGCG is present in higher levels in green tea in comparison with that of normal tea. EGCG is the component that is majorly responsible for the health benefits from green tea. It is correlated for the anticancerous property (Havsteen, 2002). The compound aids in weight loss etc.

Amino acids that are present in tea are majorly responsible peculiar umami taste of tea. Out of which the major amino acid present is L-Theanine. L- theanine is a compound that is responsible for that feeling of relaxation that one acquired when they drink tea. It also promotes brain activity. The enzymes that are present in tea include polyphenol oxidase and peroxidase which are responsible for the browning of tea leaves (Feng, 2006). Heat inhibits these enzymes that is why the green tea leaves remain green even after the entire process. They contain the pigments, chlorophyll m, carotenoids and xanthophylls. Carbohydrates in the drink account for about $11 \%$ which is for the sweetness. It contains methylxanthines such as caffeine which is a stimulant, theobromine and theophylline.

An important property that is necessary in a health drink is to have good Antimicrobial property. This would inturn result is alarming health benefits. Many health drinks are available in the commercial market, but none focus on oral health. The field has grown vastly yet many side effects exist from various health drinks. Thus the study was designed to create a health drink and to asses the Antimicrobial aspect of the same before leading to further investigations. The aim of the present study was to evaluate the antimicrobial property of the newly formulated health drink against common oral pathogen, Streptococcus mutans.

\section{MATERIAL AND METHODS}

The various components that were taken as part of the health drink are:

1) Turmeric

2) Black pepper

3) Mango peel and fruit

4) Raisins

5) Almonds.

It was ensured that none of the components were present along with preservatives or other colouring agents as it would affect the results of the present study and would ultimately result in the failure of the hypothesis. The various components were weighed out and titrated with precision using a mixer grinder to a fine homogenous powder. Mango peel and Mango fruit were sun dried for a period of 10 days to remove the water content, this would help in the shelf life of the product as well. The sample powder was prepared by weighing out $10 \mathrm{~g}$ of whole black pepper, 18g of Almonds, $15 \mathrm{~g}$ of Raisins, $10 \mathrm{~g}$ of dried and powdered mango peel and fruit along with about $1 \mathrm{~g}$ of Turmeric powder. The above said weight of different substances was obtained through various attempts of trial and error. The health drink was constituted in accordance with a previous study where the nutritional value was assessed (Gayathri and Others, 2018)

Once the health drink was constituted, it was diluted with saline in different concentrations such as $200 \mathrm{ml}$, $400 \mathrm{ml}, 800 \mathrm{ml}, 1000 \mathrm{ml}$. The Antimicrobial property was assessed against Streptococcus mutans. Nutrient Agar was incorporated with different dilutions of the health drink and incubated with colonies of Streptococcus mutans colonies, and incubated for a period of $24 \mathrm{Hrs}$ at 37 degrees Celsius. Once the incubation period was completed the culture plates were observed. The colonies were graded and further analysis is performed and the results are obtained.

\section{RESULTS AND DISCUSSION}

In the present study where the growth of the organism was assessed in the presence of the health drink at varied concentrations, it was observed that there was reduction in the growth with increase in concentration. The final growth cultures were compared with the positive and negative controls (Fig 1, Fig 2) to estimate the efficacy of the health drink. When colonies were counted it was observed that there was a significant reduction in colonies between $200 \mathrm{ml}$ of the extract and $1000 \mathrm{ml}$ of the extract (Fig 3, Fig 4) . This was a very significant finding as the usage of the health drink would inturn potentially decrease the microbial load in the oral cavity. In order to understand the exact reason for the Antimicrobial activity of the particular health drink it is important to understand each of the elements that have been used in the health drink.

The first component that was included was turmeric. Turmeric has been used through ages in various forms of medicine to treat various ailments (Prasad and Aggarwal, 
2011). When the oral cavity is being considered it has been found efficacious against various diseases such as periodontitis (Kandwal, Mamgain and Mamgain, 2015) and even in delaying the process of radiation induced mucositis in patients undergoing radiation therapy for head and neck cancers (Kandwal, Mamgain and Mamgain, 2015; David, Ruban David and Timple Shree, 2019). When the aspect of dental caries is considered, the main component of turmeric that is attributed to the activity against Streptococcus mutans is curcumin in turmeric (Song et al., 2012) as it prevents the adherence of S. Mutans to the surface of the tooth and to extracellular matrix. The first step that is involved in the formation of biofilm is bacterial adhesion and thus intervention at this stage would prevent the further development of the disease. This explains the need for turmeric in the present health drink and the reason for its Antimicrobial property in the present study can be attributed to the same.

Figure 1: Figure shows positive control with inoculation of agar with S.mutans

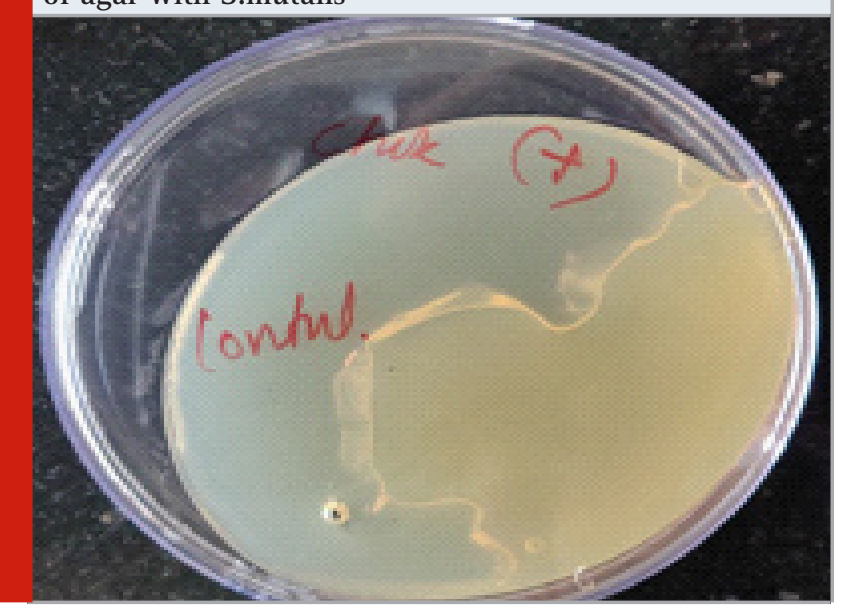

Figure 2: Figure shows negative control with inoculation of agar with saline.

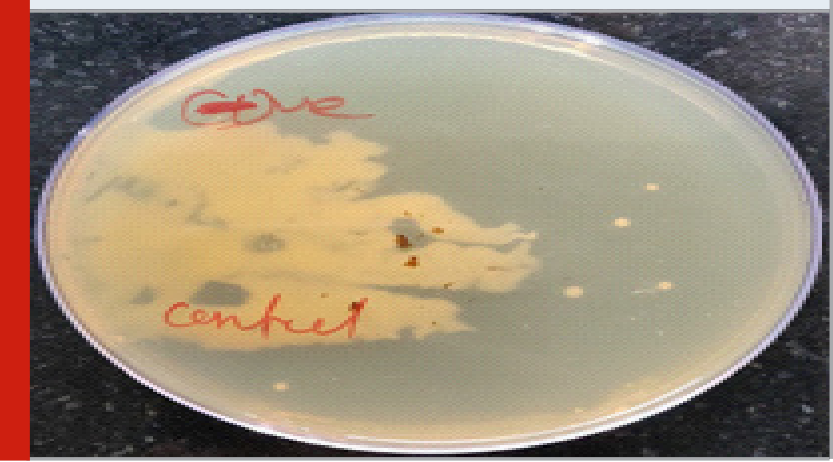

The next constituent of the health drink was black pepper. Black pepper has been attributed to various health benefits in the past including its immunomodulatory and anti cancerous properties (Majdalawieh and Carr, 2010). It has also been attributed to increase the pharmacokinetic action of certain drugs that are administered orally and are metabolised in the liver (Han, 2011). In the present study pepper has been added due to the additive property of an element that has been present in pepper known as piperine . In a study conducted by Shoba et al (Shoba et al., 1998)where the absorption of curcumin was observed in rats, it was observed that when administered in combination with piperine, the absorption rates were enhanced. Thus the addition of the same would improve the overall benefit of the health drink as well. In a study conducted by Sidarta et al (Sidarta, 2013) where pepper was tested against Streptococcus mutans it was observed to have good Antimicrobial property which is in acceptance with the findings from the present study. Thus this could also be attributed to the Antimicrobial activity of the present health drink.

The next three components that were added were mango

Figure 3: Figure shows result after incubation for 24 hours with formulated health drink with $200 \mathrm{ml}$ dilution.

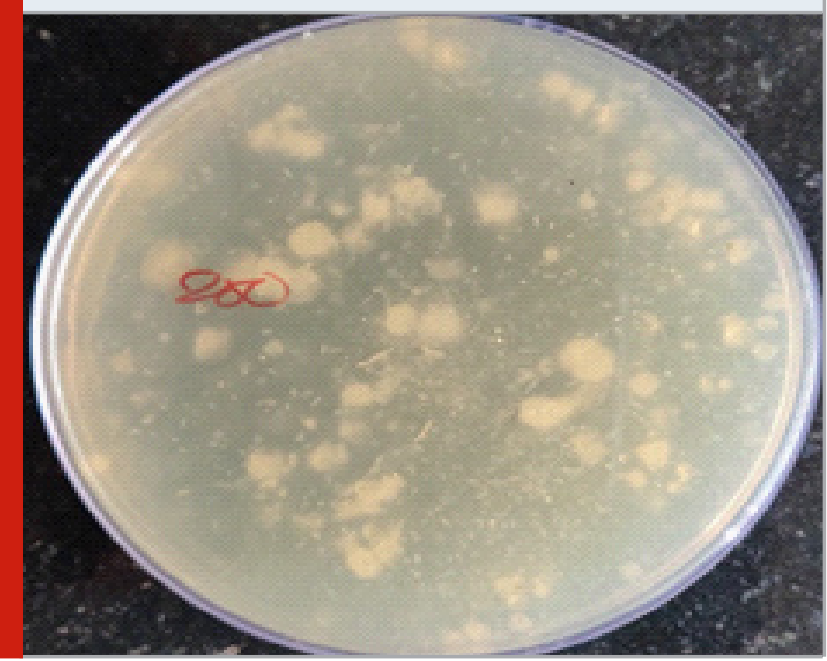

Figure 4: Figure shows result after incubation for 24 hours with formulated health drink with $1000 \mathrm{ml}$ dilution.

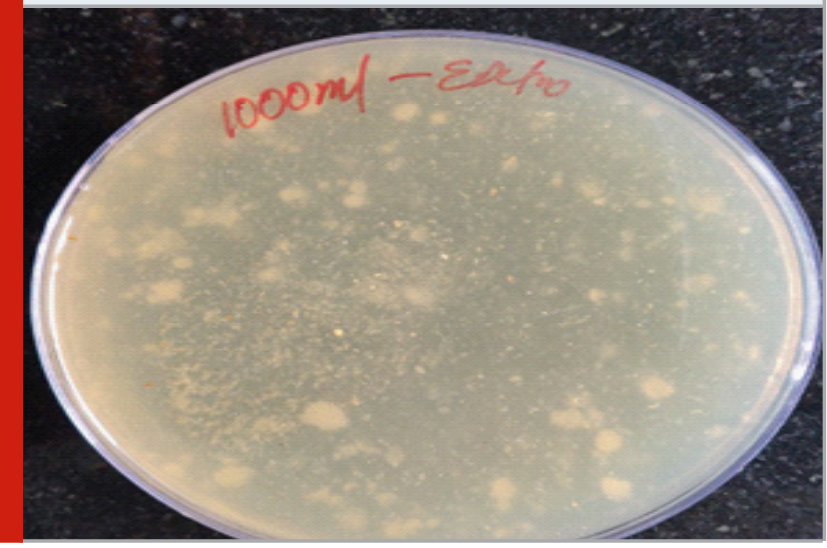

peel and mango fruit. This component was not added in the intention to attribute to the Antimicrobial property but rather for the reason of the flavonoids that are present in the mango peel and also for their dietary Fiber content which would in turn help the community (Ajila and Prasada, 2013). But surprisingly the peel of mango has also been attributed to antibacterial properties in a study conducted by Abdullah (Abdullah, 2011) . However the antibacterial property against a bacilli like streptococcus mutans was found rather less, thus it may or may not 
have contributed to the antibacterial property in the present study. But there are various other health benefits from the same and a health drink is ideally expected to satisfy all of them and not only the antibacterial property, thus the presence of the same is justified in the present health drink.

Following mango peel, the next component that was added was raisins. This is another component that was not solely added for the purpose of its Antimicrobial property but primarily for its high concentration of fluoride (Lopez and Navia, 1988)which would provide beneficial to oral health. In a study conducted by J. Fausto Rivera Cruz et al (Lopez and Navia, 1988; Clarkson and McLoughlin, 2000) it is observed that components such as oleanolic acid and butelinic acid that are present in raisins are found to be efficacious against Streptococcus mutans which is the primary oral pathogen. Thus this can also be attributed to the Antimicrobial activity of the present health drink and the presence of the same is also justified by fluoride content. Through literature it has also been reported that the same has good antibacterial activity against various other organisms such as E.coli (Greenberg, Newmann and Howell, 2005) which are notorious to cause Urinary tract infections. In a study conducted by Ramasamy Thangavelu Narendhirakannan et al (Narendhirakannan et al., 2012) where the Antimicrobial activity of various Indian herbs was assessed in rats it was observed that raisins showed a significant reduction in bacterial colonies of various species such as K. Pneumoniae and E.Faecalis. Thus the health drink would have very good systemic benefits as well.

The last component that was added in the composition is almonds, since oral health is one of the primary focuses of the formulation of the present health drink it was to be ensured that the carbohydrate concentration was kept as minimal as possible (Gayathri and Others, 2018). The sole rationale behind the addition of almonds lied in the presence of L-theanine which would inturn provide the consumer with a relaxing feeling. This is a first of its kind study where the formulation of a health drink with oral health as a priority, the usage of all these components in combination has not been reported in literature. This invitro analysis paves way for further invivo analysis to improve the health drink and bring about a positive outcome on the society with the introduction of the same. There might be certain variations between invitro and invivo analysis and hence the same has to be performed to conclude with certainty the superiority of the current health drink.

\section{CONCLUSION}

Within the limits of the present study the formulated health drink proves to have a very good antibacterial activity against common oral pathogens and the discussion also proves the efficacy of the same against systemic pathogens as well. Further studies would help further development of the health drink and introduction for consumer usage which would benefit the entire human race, one drink at a time.

\section{ACKNOWLEDGEMENTS}

We sincerely thank Saveetha Dental College for rendering full support for my study.

\section{Conflict of Interest: Nil}

\section{REFERENCES}

Abdullah, A.-S. H. (2011) 'Antibacterial activity of Malaysian mango kernel', AFRICAN JOURNAL OF BIOTECHNOLOGY. doi: 10.5897/ajb11.2746.

Ajila, C. M. and Prasada, U. J. (2013) 'Mango peel dietary fibre: Composition and associated bound phenolics', Journal of Functional Foods, pp. 444-450. doi: 10.1016/j.jff.2012.11.017.

Clarkson, J. J. and McLoughlin, J. (2000) 'Role of fluoride in oral health promotion', International Dental Journal, pp. 119-128. doi: 10.1111/j.1875-595x.2000. tb00552.x.

David, P. R., Ruban David, P. and Timple Shree, K. (2019) 'Effectiveness of Turmeric Mouthwash and Sodium Bicarbonate Mouthwash to Reduce Oral Mucositis among Patient Undergoing Radiation Therapy', Scholars International Journal of Traditional and Complementary Medicine, pp. 124-127. doi: 10.36348/sijtcm.2019. v02i07.001.

Feng, W. Y. (2006) 'Metabolism of Green Tea Catechins: An Overview', Current Drug Metabolism, pp. 755-809. doi: $10.2174 / 138920006778520552$.

Gayathri, R. and Others (2018) 'Assessment of nutritional value of a newly formulated health drink', International Journal of Research in Pharmaceutical Sciences, 9(3). Available at: https://pharmascope.org/index.php/ijrps/ article/view/265.

Glade, M. J. (2000) 'The American Association of Oriental Medicine's Complete Guide to Chinese Herbal Medicine', Nutrition, pp. 1122-1123. doi: 10.1016/ s0899-9007(00)00393-2.

Greenberg, J. A., Newmann, S. J. and Howell, A. B. (2005) 'Consumption of Sweetened Dried Cranberries Versus Unsweetened Raisins for Inhibition of Uropathogenic Escherichia coli Adhesion in Human Urine: A Pilot Study', The Journal of Alternative and Complementary Medicine, pp. 875-878. doi: 10.1089/ acm.2005.11.875.

Han, H.-K. (2011) 'The effects of black pepper on the intestinal absorption and hepatic metabolism of drugs', Expert Opinion on Drug Metabolism \& Toxicology, pp. 721-729. doi: 10.1517/17425255.2011.570332.

Havsteen, B. H. (2002) 'The biochemistry and medical significance of the flavonoids', Pharmacology \& Therapeutics, pp. 67-202. doi: 10.1016/s01637258(02)00298-X. 
Kandwal, A., Mamgain, R. and Mamgain, P. (2015) 'Comparative evaluation of turmeric gel with $2 \%$ chlorhexidine gluconate gel for treatment of plaque induced gingivitis: A randomized controlled clinical trial', AYU (An International Quarterly Journal of Research in Ayurveda), p. 145. doi: 10.4103/09748520.175537.

Knop, J. et al. (2015) 'Inhibitory Effects of Green Tea and (-)-Epigallocatechin Gallate on Transport by OATP1B1, OATP1B3, OCT1, OCT2, MATE1, MATE2-K and P-Glycoprotein', PLOS ONE, p. e0139370. doi: 10.1371/journal.pone.0139370.

Lopez, H. and Navia, J. M. (1988) 'A Method to Assay Fluoride in Foods, Beverages, and Diets', Caries Research, pp. 210-216. doi: 10.1159/000261108.

Majdalawieh, A. F. and Carr, R. I. (2010) 'In Vitro Investigation of the Potential Immunomodulatory and Anti-Cancer Activities of Black Pepper (Piper nigrum) and Cardamom (Elettaria cardamomum)', Journal of Medicinal Food, pp. 371-381. doi: 10.1089/ jmf.2009.1131.
Narendhirakannan, R. T. et al. (2012) 'Evaluation of antibacterial, antioxidant and wound healing properties of seven traditional medicinal plants from India in experimental animals', Asian Pacific Journal of Tropical Biomedicine, pp. S1245-S1253. doi: 10.1016/s22211691(12)60394-3.

Prasad, S. and Aggarwal, B. (2011) 'Turmeric, the Golden Spice', Oxidative Stress and Disease, pp. 263-288. doi: 10.1201/b10787-14.

Shoba, G. et al. (1998) 'Influence of Piperine on the Pharmacokinetics of Curcumin in Animals and Human Volunteers', Planta Medica, pp. 353-356. doi: 10.1055/s2006-957450.

Sidarta, Y. 0. (2013) 'White Pepper Extract (Piper nigrum L.) as Antibacterial Agent for Streptococcus mutans In Vitro', IOSR Journal of Dental and Medical Sciences, pp. 25-29. doi: 10.9790/0853-0462529.

Song, J. et al. (2012) 'Curcumin suppresses Streptococcus mutans adherence to human tooth surfaces and extracellular matrix proteins', European Journal of Clinical Microbiology \&t Infectious Diseases, pp. 1347-1352. doi: 10.1007/s 10096-011-1448-y. 